\title{
Childhood Nasopharyngeal Carcinoma: Review of Current Treatment Strategies
}

\author{
Encheva E. ', Ivanovska Hr. ${ }^{1}$, Iliev G. ${ }^{2}$ \\ 'Radiotherapy Department, University Hospital "St. Marina" - Varna \\ 2ENT Department, University Hospital "St. Marina" - Varna
}

\begin{abstract}
Childhood Nasopharyngeal carcinoma (NPC) is a rare tumor among children, associated with Epstein-Barr virus infection andmore advanced disease at presentation, usually undifferentiated carcinoma. The distant failure is common observed among childhood NFC, which implies the need of early systemic treatment, but radiotherapy still is the main pillar of treatment. As the children are still growing organism, the cure of the patients is at the cost of late morbidity. The use of advanced radiotherapy techniques like IMRT (Intensity modulated radiotherapy) is beneficial for better sparing of surrounding organ at risk and reducing toxicity. Most common protocol used in European patients nowadays is NPC-2003- GPOH (German Pediatric Oncology Hematology Society), which has shown the best treatment results compared with other pediatric NPC study groups even with the strategy to deescalate the radiation dose in good responders to induction chemotherapy. However international randomize control trials are needed to answer when is safe to deescalate the radiation dose, could concomitant chemotherapy be omitted in case of good response of the induction chemotherapy, and is the maintenance therapy with interferon beta needed in all patients.
\end{abstract}

Key words: Childhood nasopharyngeal carcinoma, treatment, radiotherapy, deescalation radiation dose, chemoradiation, VMAT

\section{Introduction}

Childhood Nasopharyngeal carcinoma (NPC) is a rare tumor among children and accounts only 1$5 \%$ of pediatric malignancy [1]. Higher incidence is seen in Asian countries (2-2.5 /million population) compared to USA (0.1-1.5/million) [1]. Male children are more often affected with median age of occurrence 13 years [1]. NPC is associated with Epstein-Barr virus infection andmore advanced disease at presentation with usually WHO type III, undifferentiated carcinoma. The distant failure is common observed among childhood NFC, which implies the need of early systemic treatment [2, 3] However radiotherapy still is the main pillar of treatment [4]. As the children are still growing organism, the cure of the patients is at the cost of late morbidity $[4,5]$.

Clinical manifestation of NPC is quite diverse. Most common symptom in the patients is enlarged cervical lymph nodes unilaterally or bilaterally, which could be also painful. Typical complaints of the patients are nasal obstruction, nasal bleeding, headache, hearing lost, tinnitus, double or lost vision, swallowing problems, and symptoms caused by cranial nerves involvement in case of scull base invasion. 
According to WHO three subtypes of NPC are defined. Type I is keratinising squamous cell carcinoma,type II is a non-keratinising epidermoid carcinoma,and type III is undifferentiated carcinoma, very oftencalled lymphoepithelioma of Schminke [6].

ENT endoscopic evaluation with biopsy is needed to histologically prove the diagnosis of NPC.

\section{Imaging}

In the era of modern imaging technologies we could rely to better stage the tumor for distant metastasis and detect early progression with PET/ CT [7]. FDG PET/CT in adults has proven its role for regional lymph node involvement with high accuracy rate and sensitivity of $97 \%-100 \%$ and a specificity of $73 \%-97 \%$. For distant spread, FDG PET/CT is more accurate compared with conventional CT. There is only one study in children evaluating the role of PET/CT for staging and follow-up in 18 cases. For staging a better concordance between MRI and PET/CT in N stage was seen than in T stage. For post treatment follow-up, the concordance was $100 \%$ at 9 months [7].

The Pediatric German group (GPOH), recommends PET/CT for staging, restaging and follow up despite a low level of evidence in childhood NPC, because the high incidence of locoregionally advanced cancer in children, for precisely detection of involved lymph nodes (important for radiotherapy volumes), and $\mathrm{M}$ staging [8].

MRI still is the most sensitive imaging method for evaluation of local tumor extension, local tumor growth, skull-base involvement, and nodal metastasis.MRI application give us better information on the primary tumor extension intracranialy and itraorbitally. The better the staging the most appropriate treatment could be used and this an improved treatment result is expected.

\section{Radiotherapy}

NPC is well known for its radiosensitivity, which is the reason radiotherapy to be the main treatment modality for decades. Traditionally the total radiation dose delivered to the primary tumor and the involvedlymph nodes range between 66 and 70Gy, and for the uninvolved regional lymph node drainage between 46 and 50Gy. Radiotherapy alone in locoregional advanced NPC achieved only $20-40 \%$ 5 year survival [1] with significant early and late morbidity [9].

\section{Radiation dose}

The correlation between radiation dose and OS and local control was studied. The optimal radiotherapy dose is not defined yet, especially when radiotherapy is delivered in combinationwith chemotherapy. Some authors $[10,11]$ found improvement of the local control withhigher total dose. Higher radiation dose in children correlated with significant late term morbidity $[12,13]$. No added benefit in terms of survival was observed between patients treated with high (forprimary tumor total dose higher than 68 Gy and forregional lymph node higher than 60 Gy) and low radiation dose (primary tumor $68 \mathrm{~Gy}$, regional lymph nodes 60Gy) [5, 14]. Strategies for reducing the radiation dose with maintaining the tumor control are explored. The first prospective study reducing the radiation dose was the POG study 9486 [3] with total dose of 61.2 Gy to the primary tumor and involved lymph nodes after four cycles of induction chemotherapy (methotrexate, cisplatin, 5-fluorouracil, and leucovorin). The reported4-year event free survival and overall survival rats were $77 \%$ and $75 \%$, suggesting that total radiation dose below60 $\mathrm{Gy}$ is reasonable. Two more prospective trials of NPC- 91-German Society of Pediatric Oncology and Hematology and NPC-2003-German Society of Pediatric Oncology and Hematology /Dutch Childhood Oncology Group studied deescalation or radiotherapy dose after induction chemotherapy, boosted by addition of IFN beta[2, 15]. They implemented total radiation dose to $59.4 \mathrm{~Gy}$, which further was decreased to $50 \mathrm{~Gy}$ in full responders to induction chemotherapy. This study protocol reached the best reported results in a matter of overall survival and event free survival for a median follow up period of 30 and 48 months (95-97\% and 91- 92.4\%). The Curie Institute in Paris reduced the cervical lymph nodes dose to less than $50 \mathrm{~Gy}$, in the good responders with more than $90 \%$ decrease of tumor volume. In $50 \%$ of the patients reduction of lymph node irradiation dose was done with a similar regional failure $[3,16]$.

\section{Radiotherapy techniques}

In order to reduce early and late morbidity the use of modern radiotherapy technologies is beneficial $[10,17]$. Better sparing of surrounding organ at risk is possible with implementation of advanced radiotherapy techniques like IMRT. 
The application of of IMRT in pediatric NPC was studied by few authors.Conventional 2D (dimensional) radiation therapy was compared with IMRT (Intensity Modulated Radiotherapy) in 34 children with NPC, and the recurrence free survival was improved with IMRT use $(84.21 \%$ vs $68.28 \%)$, although statistical significance was not proved, because the small patients cohort [10]. Grade 3 toxicity was observed less common in the IMRT arm. Other authors investigated the use of IMRT with SIB (simultaneous integrated boost) in 34 patients and also found reduced toxicity and excellent 5-year local recurrence free survival, distant metastasis free survival,disease free survival, and overall survivalas follows $97.1 \%, 88.2 \%, 85.3 \%$, and $88.2 \%$, [17]. Recent study has reported 95 children and adolescents treated with IMRT to achieve 4-year overall survival, locoregional relapse free survival, progression free survival, and distant metastasis free survival respectively $90.8 \%, 94.9 \%, 79.1 \%$, and $84.0 \%$, with relatively low percent of grade 3 and 4 toxicity respectively $20 \%$ for mucositis, $4.2 \%$ for skin toxicity, and $14.7 \%$ for bone marrow suppression [5]. Grade 4 toxicity was observed only in the group of concomitant chemoradiation. In another 2 studies IMRT was used in more than $30 \%$ of the cases with the 5 -year OS about $80 \%$ but treatment toxicities was not reported $[18,14]$. Proton therapy also reduces the dose at normal tissue and decreases the late morbidity in childhood NPC [19].

\section{Chemotherapy}

As radiotherapy was the leading treatment modality, the chemotherapy treatment was reserved for metastatic stage of recurrences. First was explored treatment with cyclophosphdmide alone, than in combination with different cytostatics and finally the current standard platinum based therapy was implemented [16]. Distant failure was often observed in children, which led to introducing the induction chemotherapy followed by radiation or chemoradiation $[2,3]$. In $80 \%$ of the children the induction therapy achieved more than 50\% response [16]. Another idea behind the induction chemotherapy is to help to decrease the radiation dose and respectively to reduce the early and late morbidity

\section{Current treatment protocols}

The evolution of childhood NPC went from solely radiotherapy through combining chemotherapy with radiotherapy and finally adding immunotherapy like IFN beta (interferon beta). Undifferentiated nasopharyngeal carcinoma is highly sensitive to external beam radiotherapy, and for this reason is the main part of multimodality strategy [1]. Radiotherapy alone showed survival rates of $20-60 \%$ at five years $[1,16]$.Most common protocol used in European patients nowadays is NPC-2003- GPOH, which shows the best results compared with other pediatric NPC study groups with an event-free survival rate of $92.4 \%$ and overall survival rate of $97.1 \%$ at a median follow-up of 30 months [15]. All patients received 3 coursed induction chemotherapy, followed by concomitant cisplatin during the first week and last week of irradiation. After irradiation, all patients received IFN beta for 6 months. From the new century induction chemotherapy in combination with chemoradiation were evaluated in 6 prospective studies (from 18 to 111 childhood NPC) $[2,3,14,15,20,21]$. All of them reported very good results with best achieved from the German study group [15].But still no randomized clinical trial has compared the concomitant chemoradiotherapy versus induction chemotherapy and concomitant chemoradiotherapy. It is not clear yet if induction or concurrent chemotherapy has bigger contribution for improvement of treatment results, as both were implemented in clinical practice at once. But an induction chemotherapy is recommended in childhood locoregionally advanced NPC in order to prevent distant failure, to select the good responders, where deescalated radiotherapy could be used, and to deliver radiation therapy in patients with better performance status. In patients irradiated with IMRT, the contribution of concurrent chemotherapy is still under investigation. Three studies reported no added treatment benefit in a matter of overall survival, disease free survival or local control with adding concurrent chemotherapy when IMRT technique was used $[22,23]$. Phase III clinical trials are expected to better define the role of concurrent chemotherapy in patients treated with IMRT.

\section{Prognostic markers}

The reported recurrence and distant metastasis rate is about $20-50 \%$ in the first or second year of diagnosis; the prognosis in this patients is poor with survivals only among the recurrent cases [6]. Many studies proved that $\mathrm{T}$ category is not reliable prognostic factor for freedom of local recurrences 
$[5,24]$, but $\mathrm{N}$ classification is considered independent unfavorable prognostic factor $[22,25]$. In a recent study $\mathrm{N}$ category proved to be the prognostic factor that could influence the freedom from distant metastasis $(p=0.54)$ [22]. Fourteen patients in that study showeddistant failure, with a median time of 11 months (range 5-44 months), like in 13 patientsit was within 2 years after radiotherapy. Bones were more often affected (12 patients), followed by lung (4 patients), and liver ( 2 patients).

Age also is considered prognostic factor. Better overall and event free survival is reported in NPC diagnosed below age of 14 compared to age 14 to 18 [26].

The serum level of Epstein-Barr virus DNA infection could serve also as prognostic and predictive marker. Theunderstanding of the pathogenic role of Epstein-Barr virus led to explore different treatment strategies [6]. In the studies of German Society of Pediatric Oncology and Hematology, IFN beta was given for a 6-month of maintenance therapy after standard chemoradiotherapy regimen to increase immune system capability to detect cells carriers of the Epstein-Barr virus and eradicate any persistent tumor cells resistant to radiotherapy and chemotherapy. Using this treatment strategy the best overall survival results were reached[2, 15].

\section{Conclusion}

The current status of the data supports the implementation of multimodality imaging for staging childhood NPC. The recently established treatment protocol in locoregionaly advanced stages induction chemotherapy followed by chemoradiation and interferon beta maintenance therapy contributes to superior overall survival (higher than $85 \%$ ) compared to radiotherapy alone (30-60\%). Acute and late morbidity are still a concern and ways of improvement are investigated like IMRT and dose deescalation in good responders to induction chemotherapy. International randomize control trials are needed to answer when is safe to deescalate the radiation dose, could concomitant chemotherapy be omitted in case of good response of the induction chemotherapy, is the maintenance therapy needed in all patients.

\section{Bibliography:}

[1] Ayan I, Altun M. Nasopharyngeal carcinoma in children: retrospective review of 50 patients. Int J Radiat Oncol Biol Phys. 1996;35:485492.

[2] Mertens R, Granzen B, Lassay L, et al. Treatment of nasopharyngeal carcinoma in children and adolescents: definitive results of a multicenter study (NPC-91-German Society of Pediatric Oncology and Hematology ). Cancer 2005;104:1083-1089.

[3] Rodriguez-Galindo C, Wofford M, Castleberry RP, et al. Preradiation chemotherapy with methotrexate, cisplatin, 5-fluorouracil, and leucovorin for pediatric nasopharyngeal carcinoma. Cancer 2005;103:850-85

[4] Dourthe ME, Bolle S, Temam S, Jouin A, Claude L, Reguerre Y, Defachelles AS, Orbach D, Fresneau B. Childhood Nasopharyngeal Carcinoma: State-of-the-Art, and Questions for the Future. J Pediatr Hematol Oncol. 2018 Mar;40(2):85-92

[5] Guo Q, Cui X, Lin S, Lin J, Pan J Locoregionally advanced nasopharyngeal carcinoma in childhood and adolescence: Analysis of 95 patients treated with combined chemotherapy and intensity-modulated radiotherapy.Head Neck. 2016 Apr;38 Suppl 1:E665-72.

[6] Ayan I, Kaytan E, Ayan N. Childhood nasopharyngeal carcinoma: from biology to treatment. Lancet Oncol 2003;4:13-21

[7] Cheuk DKL, Sabin ND, Hossain M, et al. PET/CT for staging and follow-up of pediatric nasopharyngeal carcinoma. Eur J Nucl Med Mol Imaging. 2012;39:1097-1106

[8] Kontny U, Franzen S, Behrends U, et al. Diagnosis and treatment of nasopharyngeal carcinoma in children and adolescents - recommendations of the GPOH-NPC study group. Klin Padiatr. 2016;228:105-112

[9] Uzel O, Yörük SO, Sahinler I, et al. Nasopharyngeal carcinoma in childhood: long-term results of 32 patients. Radiother Oncol J Eur Soc Ther Radiol Oncol. 2001;58:137-141.

[10] Laskar S, Bahl G, Muckaden M, et al. Nasopharyngeal carcinoma in children:comparison of conventional and intensity-modulated radiotherapy. Int J Radiat Oncol Biol Phys 2008;72:728-736.

[11] Wolden SL, Steinherz PG, Kraus DH, Zelefsky MJ, Pfister DG, Wollner N. Improved long-term survival with combined modality therapy for pediatric nasopharynx cancer. Int J Radiat Oncol Biol Phys 2000;46:859-864.

[12] Ozyar E, Selek U, Laskar S, et al. Treatment results of 165 pediatric patients with non-metastatic nasopharyngeal carcinoma: a Rare Cancer Network study. Radiother Oncol 2006;81:39 46

[13] Cheuk DK, Billups CA, Martin MG, et al. Prognostic factors and long-term outcomes of childhood nasopharyngeal carcinoma. Cancer 2011;117:197- 206

[14] Casanova M, Bisogno G, Gandola L, et al. A prospective protocol for nasopharyngeal carcinoma in children and adolescents: the Italian Rare Tumors in Pediatric Age (TREP) project. Cancer 2012;118:2718-2725.

[15] Buehrlen M, Zwaan CM, Granzen B, et al. Multimodal treatment, including interferon beta, of nasopharyngeal carcinoma in children and young adults: preliminary results from the prospective, multicenter study NPC-2003-GPOH/DCOG. Cancer. 2012;118:4892-4900 
[16] Orbach D, Brisse H, Helfre S, et al. Radiation and chemotherapy combination for nasopharyngeal carcinoma in children: radiotherapy dose adaptation after chemotherapy response to minimize late effects. Pediatr Blood Cancer 2008;50:849-853.

[17] Tao CJ, Liu X, Tang LL, et al. Long-term outcome and late toxicities of simultaneous integrated boost-intensity modulated radiotherapy in pediatric and adolescent nasopharyngeal carcinoma. Chin J Cancer 2013;32:525-532.

[18] Yan Z, Xia L, Huang Y, Chen P, Jiang L, Zhang B. Nasopharyngeal carcinoma in children and adolescents in an endemic area: a report of 185 cases. Int J Pediatr Otorhinolaryngol 2013;77:1454-1460

[19] Oshiro Y, Sugahara S, Fukushima T, et al. Pediatric nasopharyngeal carcinoma treated with proton beam therapy. Two case reports. Acta Oncol 2011;50:470-473.

[20] Rodriguez-Galindo C, Krailo MD, Krasin MJ, et al. Treatment of childhood nasopharyngeal carcinoma (cNPC) with neoadjuvant chemotherapy (NAC) and concomitant chemoradiotherapy(CCRT): Results of the Children's Oncology Group ARAR0331 study. J Clin Oncol. 2016;34:10513.

[21] Casanova M, Özyar E, Patte C, et al. International randomized phase 2 study on the addition of docetaxel to the combination of cisplatin and 5-fluorouracil in the induction treatment for nasopharyngeal carcinoma in children and adolescents. Cancer Chemother Pharmacol. 2016;77:289-298.

[22] Lin S, Lu JJ, Han L, Chen Q, Pan J. Sequential chemotherapy and intensity-modulated radiation therapy in the management of locoregionally advanced nasopharyngeal carcinoma: experience of 370 consecutive cases. BMC Cancer 2010;10:39.

[23] Sun X, Su S, Chen C, et al. Long-term outcomes of intensity-modulated radiotherapy for 868 patients with nasopharyngeal carcinoma: an analysis of survival and treatment toxicities. Radiother Oncol 2014;110:398-403.

[24] Wong FC, Ng AW, Lee VH, et al. Whole-field simultaneous integratedboost intensity-modulated radiotherapy for patients with nasopharyngeal carcinoma. Int J Radiat Oncol Biol Phys 2010;76:138-145.

[25] Lin S, Pan J, Han L, et al. Update report of nasopharyngeal carcinoma treated with reduced-volume intensity-modulated radiation therapy and hypothesis of the optimal margin. Radiother Oncol 2014;110:385-389

[26] Saeed H, Zaidi A, Adhi M, et al. Pediatric nasopharyngeal carcinoma: a review of 27 cases over 10 years at Shaukat Khanum Memorial Cancer Hospital and Research Center, Pakistan. Asian Pac J Cancer Prev. 2009;10:917-920. 